\title{
Improved Low Energy Adaptive Clustering Hierarchy in Wireless Sensor Network Routing Protocols
}

\author{
Ahmed O. Eid ${ }^{\# 1}$, Amr Zamel ${ }^{* 2}$, Ibrahim E. Ziedan ${ }^{\# 3}$ \\ ${ }^{\#}$ Computer and Systems Dept. Faculty of Engineering Zagazig University \\ Computer and Systems Dept. - Faculty of Engineering - Zagazig University- Egypt \\ a7med_3osman@yahoo.com \\ 3 ieziedan@gmail.com \\ * Computer and Systems Dept. Faculty of Engineering Zagazig University \\ Computer and Systems Dept. - Faculty of Engineering - Zagazig University- Egypt \\ 2 eng.amrzml@gmail.com
}

\begin{abstract}
In recent years, applications of wireless sensor networks cover a wide range of different fields as industrial, agriculture, military and many others. As the resources of nodes of wireless sensor network (WSNs) are limited, improvement of wireless sensor network routing protocols has become the destination of many researchers. Many researches try to solve problems of wireless sensor network, especially the life time problem, using meta-heuristic algorithms. This paper introduces an improved lowenergy adaptive clustering hierarchy (MLEACH). MLEACH suggests an equation for the selection of cluster heads based on the sine-cosine optimization algorithm. Simulation results show that MLEACH has improvements of network lifetime as well as its energy efficiency.
\end{abstract}

Keyword - Wireless Sensor Network, Routing Protocols, LEACH, Threshold, Meta-heuristic and SineCosine Algorithm

\section{INTRODUCTION}

At present smart environment in industry, buildings, agriculture and transportation systems depends on Wireless Sensor Networks (WSNs) to collect data [1]. WSNs act may as infrastructures for usual networks; each consists of a group of smart nodes with limited energy and small capability of processing. Such nodes have two main functions. The first is sensing the physical parameters or variables that the network is established for according to its application such as temperature, light, earth vibrations and sound. The second function is gathering data, analyzing them and then sends such data to the base station that is responsible for taking decisions based on the information received. In the last two decades the field of optimization algorithms has noted significant developments[2, 3]. Many researches investigated the problem of wireless sensor routing protocols so as to decrease the energy consumption of routing information from nodes to the base station aiming to prolong the lifetime of the network. Heizelman developed and implemented the first distributed and clustered routing protocol with low energy consumption called LEACH (Low Energy Adaptive Clustering Hierarchy) [4]. Then other improvements were introduced based on LEACH [5, 6]. This paper introduces an improvement to LEACH routing protocol based on a meta-heuristic approach. Optimization algorithms are discussed to find the optimal values for parameters of a given system. Optimization problems can be found in all research topics that make such algorithms very essential for researchers.

\section{RELATED WORK}

Xiangning and Yulin proposed an algorithm that improves the performance of LEACH routing protocol. The algorithm proposes a method for the choice of cluster heads, based on the energy of the nodes. Also the communication mode is changed from a single hob to a multiple hob one from a cluster head to the base station [7].

Ali, Dey, and Biswas proposed an algorithm called ALEACH that improves the selection of cluster heads in LEACH. The algorithm selects the best node to be a cluster head based on the principle of distributing energy load among all the nodes [8].

Wang, Du and Xu introduced a LEACH-TM algorithm that is based on the principle of trust, they proposed a cluster-head adjusting procedure that makes the cluster heads like routers having a multi-path between the cluster heads and the base station. This principles makes the performance of LEACH more efficient and increases the lifetime of the network [9]. 
$\mathrm{Xu}$, Jin and Lou proposed an algorithm called ELEACH. The way of selection of a cluster head in LEACH is random and the round time is fixed. The algorithm takes the remnant power of sensor nodes in to consideration so as to balance the network energy load and change the round time based on an optimal cluster size. This improvement of LEACH increases the lifetime of the network at least by $40 \%$ [10].

Katiyar, Chand and Gautam introduced an energy efficient clustering protocol (FZ-LEACH). its algorithm eliminates the problem of existence of very large and very small clusters at the same time. It solves the problem by using the concept of Far-Zone. Far-Zone is a group of sensor nodes in the network whose energies is less than a threshold value [11].

\section{Sine-Cosine OPTIMIZATION ALgORITHM}

A sine-cosine algorithm is developed for solving the optimization problem. It depends on creating a number of random solutions and then using a mathematical model based on sine-cosine function to find the best solution for the problem. Several random and adaptive variables are integrated together in the sine-cosine algorithm so as to make sure of the exploration and exploitation of the search space during the search for the global solution. The sine-cosine algorithm performance is measured in three phases. The first phase is performed using wellknown test cases to test the exploration and exploitation, local optimum solution avoidance and convergence of the algorithm. The second phase uses some other metrics such that the search history, the average fitness of each solution and the best solution from the search space is obtained. Thirdly, the application of such algorithm has some difficulties to verify its performance in solving problems. The sine-cosine algorithm is described through the following steps

I. Initialize a set of random solutions.

Do

II. Evaluate the fitness of each solution by appling the fitness function.

III. Update the best solution.

IV. Update r1,r2,r3,r4 that are random values between 0 and 1.

V. Update the search agents using the following equations,

$$
X_{i}^{j+1}=\left\{\begin{array}{l}
X_{i}^{j}+r_{1} \times \sin r_{2} \times\left|r_{3} p_{i}^{j}-X_{i}^{j}\right|, r_{4}<0.5 \\
X_{i}^{j}+r_{1} \times \cos r_{2} \times\left|r_{3} p_{i}^{j}-X_{i}^{j}\right|, r_{4} \geq 0.5
\end{array}\right.
$$

where $\mathrm{t}$ (iteration number) $<$ maximum number of iterations

VI. Return the best solution obtained by the algorithm.

The above pseudo code shows that the algorithm starts with a set of random solutions. Then the algorithm saves the best solution after calculating the fitness functions. The algorithm then updates the random values and search agents. These steps are repeated until the maximum number of iteration is completed then it returns the best solution [12].

\section{LEACH Routing PROTOCOL}

The main techniques of LEACH routing protocol include algorithms for distributing cluster head formation, adaptive cluster formation, and algorithms for selecting cluster head positions. The algorithm of distributing cluster head formation ensures self-organization of the base station. The algorithm of selecting cluster head positions and cluster formation is used to ensure that the energy dissipated is fairly shared between all nodes to prolong the lifetime of the network [7].

1) LEACH protocol Description: LEACH routing protocol works on a round concept where many rounds are run. Each round consists of two basic steps. The first is a cluster setup step and the other is a steady-state step. The first step selects the cluster heads and the steady-state step sends data from sensor nodes to base stations. Figure (1) shows the flow chart of LEACH routing protocol and its steps.

2) In the setup state LEACH selects the cluster heads from network nodes. Selecting a cluster head is based on a probability concept such that the number of cluster heads $\mathrm{K}$ in each round is a specific one. In each round, each node generates a random value between 0 and 1 and this number is compared with a time-varying threshold T (n).The node is elected to be a cluster head if its generated number is below the threshold T (n). At a cluster round $\mathrm{r}$ the threshold $\mathrm{T}(\mathrm{n})$ is described by eqn. (2) as follows.

$$
\mathbf{T}(\mathbf{n})=\left\{\begin{array}{l}
\frac{\mathbf{p}}{1-\mathbf{p}\left(\operatorname{rmod}\left(\frac{1}{\mathrm{p}}\right)\right)} \\
0
\end{array}\right.
$$

if $\mathbf{n} \in \mathbf{G}$

\section{otherwise}


Where $\mathrm{p}$ is the desired percentage of cluster heads to be chosen in each round. The maximum number of cluster heads in each round is $\mathrm{K}$. Where the number of rounds in LEACH is determined by N/K. where $\mathrm{N}$ is the number of nodes and $\mathrm{K}$ is the expected number of clusters in each round. $\mathrm{G}$ is the set of nodes that are not to be cluster heads in $1 / p$ rounds, and $r$ is the number of rounds. Once the cluster-head nodes are selected, the network starts the second step of the protocol i.e. the steady-state, step where data is sent from nodes to the base station using CSMA protocol [13]. The value of $\mathrm{p}$ is determined based on the area of the network and the number of nodes in it.

\section{THE PROPOSED AlgorithM (MLEACH)}

3) The MLEACH stands for Modified LEACH. The modification of LEACH is in the set-up step i.e. in the selection process of cluster heads. MLEACH selects cluster heads in each round based on the sine-cosine optimization algorithm. In each round as in LEACH a random number $(\mathrm{Rn})$ between 0 and 1 is generated. Also in each round, another random number $\mathrm{r} 2$ is also generated between 0 and 1 , where a threshold equation is determined based on the value of $\mathrm{r} 2$ as given by eqn. (3) where the modified equation for determining the threshold is as follows.

$$
\mathbf{T}(\mathbf{n})= \begin{cases}\mathbf{e} * \sin \mathbf{r} 1 *|\mathbf{e} * \mathbf{p} * \sin \mathbf{r}|, & r 2<0.5 \\ \mathbf{e} * \cos \mathbf{r} \mathbf{1} *|\mathbf{e} * \mathbf{p} * \cos \mathbf{r}|, & r 2 \geq 0.5\end{cases}
$$

Where $\mathrm{r} 1$ is a random value between 0 and 1 , e is the energy of the node and $\mathrm{r}$ is the number of rounds. If the generated number $(\mathrm{Rn})$ by the node is less than the threshold value $T(n)$ it will be a cluster head as the basic equation of LEACH does. The following figure (2) a and b showed the different ways for cluster head selection decision in each algorithm..

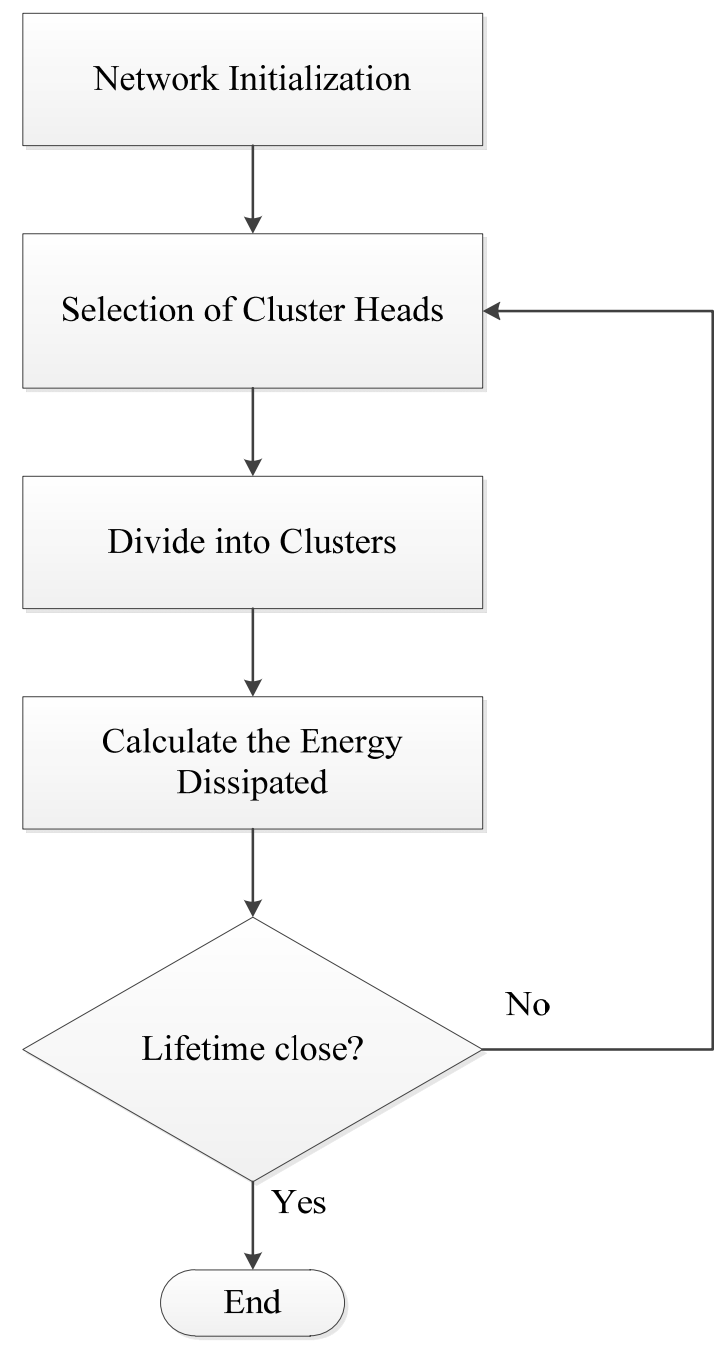

Fig (1) Flow Chart of LEACH Routing Protocol 


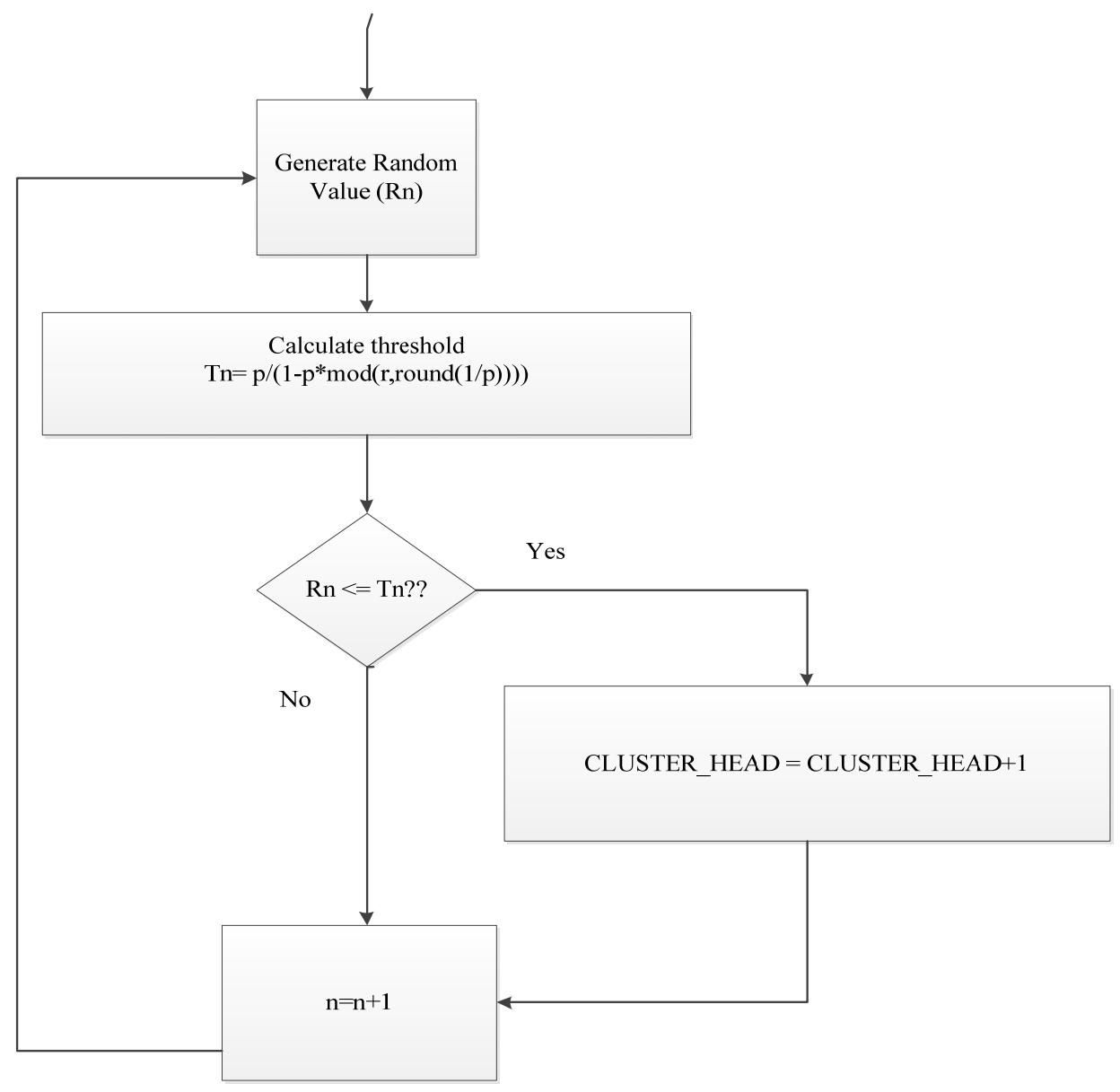

Fig (2a) Selection Cluster Head in LEACH

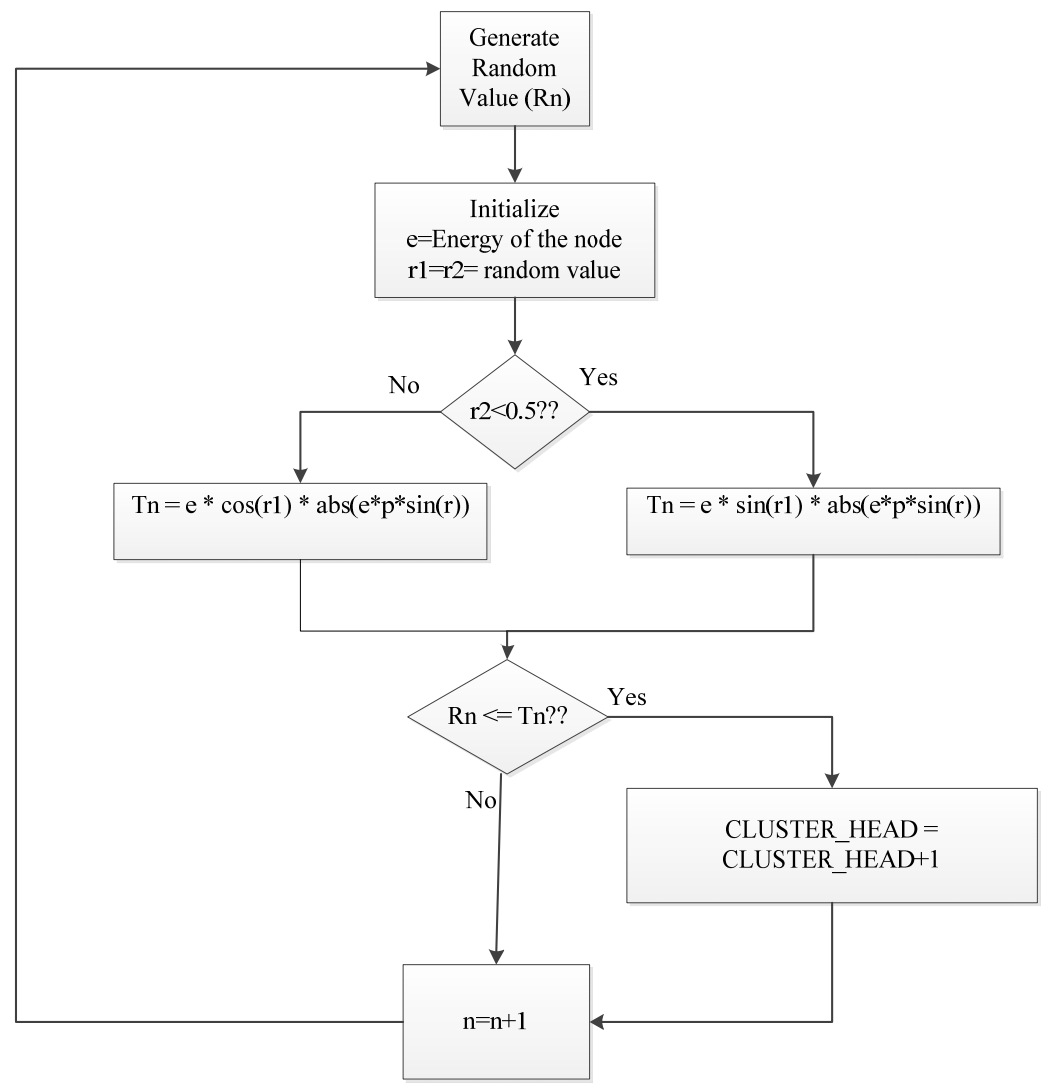

Fig (2b) Selection Cluster Head in MLEACH 


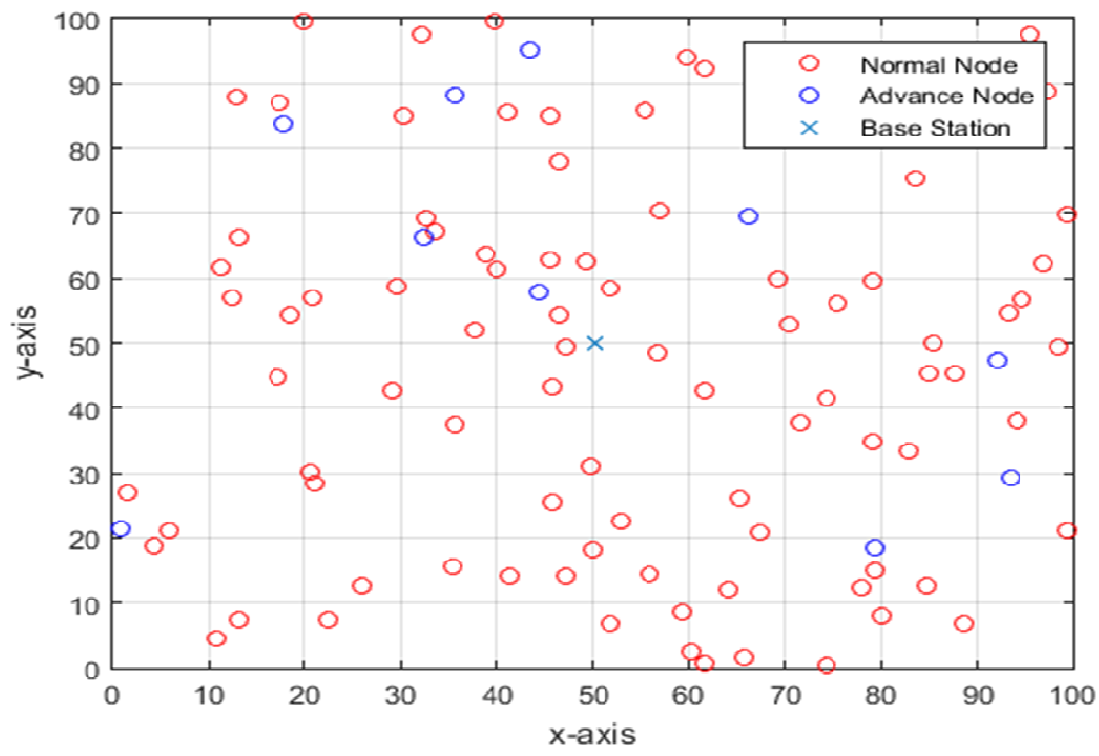

Fig (3) Network dimensions and the location of the base station

\section{TERMination CRITERIA}

Many definitions were introduced for calculating the lifetime of a wireless sensor network. The lifetime may be calculated until the first node of the network dies, or until the last node of the network dies, or until the a certain percentage of nodes die [14]. Other researches consider the lifetime of the network as the period until the entire region is covered [15]. Other researches have defined many metrics for the life time of the network as N-of-N lifetime, K-of-N lifetime and M-in-K-of-N lifetime. N-of-N lifetime means the period until the first cluster head node dies. K-of-N lifetime means the period is as long as the number of gateway nodes $\mathrm{K}$ of $\mathrm{N}$ gateways is alive. $\mathrm{M}$-in-K-of-N lifetime means the time duration until all $\mathrm{M}$ supporting gateways and overall a minimum of $\mathrm{K}$ gateways are alive [16]. In this paper an $\mathrm{N}$-of-N lifetime is considered.

\section{VII.SIMULATION RESULTS}

This section presents the simulation results. The simulation program is developed by MATLAB 2015 to analyze the network lifetime for WSNs. These results considered the lifetime of network is that until the first node of the network dies. The area of the network is $100 * 100 \mathrm{~m} 2$ and the number of sensor nodes is 100 that are randomly located in the area. Firstly, the base station is located at the center of the network as in figure (3). The initial energy for each node is $0.5 \mathrm{~J}$ and the other simulation parameters are as given in table 1.

Higher number of rounds means an increase of life time of the network. Figure (4) shows that the MLEACH (proposed algorithm) has higher number of rounds. Less energy consumption during a certain number of rounds which means also increasing the lifetime of the network. Figure (5) shows that the MLEACH has less energy consumption as compared with LEACH where the energy is measured during 100 rounds.

Secondly the location of base station in the proposed algorithm is changed to study its effect. According to the application of the WSN the base station may be located. In some applications as forest fire detection or earthquake detection the base station is preferred to be at the origin i.e. outside the area covered by the network. In other applications as agricultural estates or animal husbandry the location of base station is preferred at the center of the network. So the location of the base station was changed as in figure (6). The results showed that the proposed algorithm (MLEACH) has a number of rounds more than the LEACH routing protocol in different locations i.e. it prolongs the life time of the network in any location of base station as shown in figure (7).

It is noted from figures (6) and (7) that the number of rounds are nearly the same for the base station symmetrical location from the center point of the area covered by the network i.e. $(50,50)$. For example the number of rounds for the location $(25,50)$ is close to that of the location $(75,50)$ and so on. This may be understood in view of the symmetry in sending data to the base station regarding the number of hops. 
TABLE I. Simulation parameters

\begin{tabular}{|l|l|}
\hline Parameter & Value \\
\hline Network Size & $100 * 100 \mathrm{~m} 2$ \\
\hline Base Station Location (x, y) & $(50,50)$ \\
\hline Initial Energy for Each Node & $0.5 \mathrm{~J}$ \\
\hline P (Cluster Head Election Probability) & 0.1 \\
\hline ETX (Energy for transmit data) & $50 \mathrm{~nJ}$ \\
\hline ERX (Energy for receive data) & $50 \mathrm{~nJ}$ \\
\hline EFS (Transmit Amplifier Energy in free space) & $10 \mathrm{~nJ}$ \\
\hline EMP ((Transmit Amplifier Energy in multi-path) & 0.013 PJ \\
\hline EDA (Data Aggregation Energy) & $5 \mathrm{~nJ}$ \\
\hline M (Heterogeneity) & 0.1 \\
\hline alpha & 1 \\
\hline
\end{tabular}

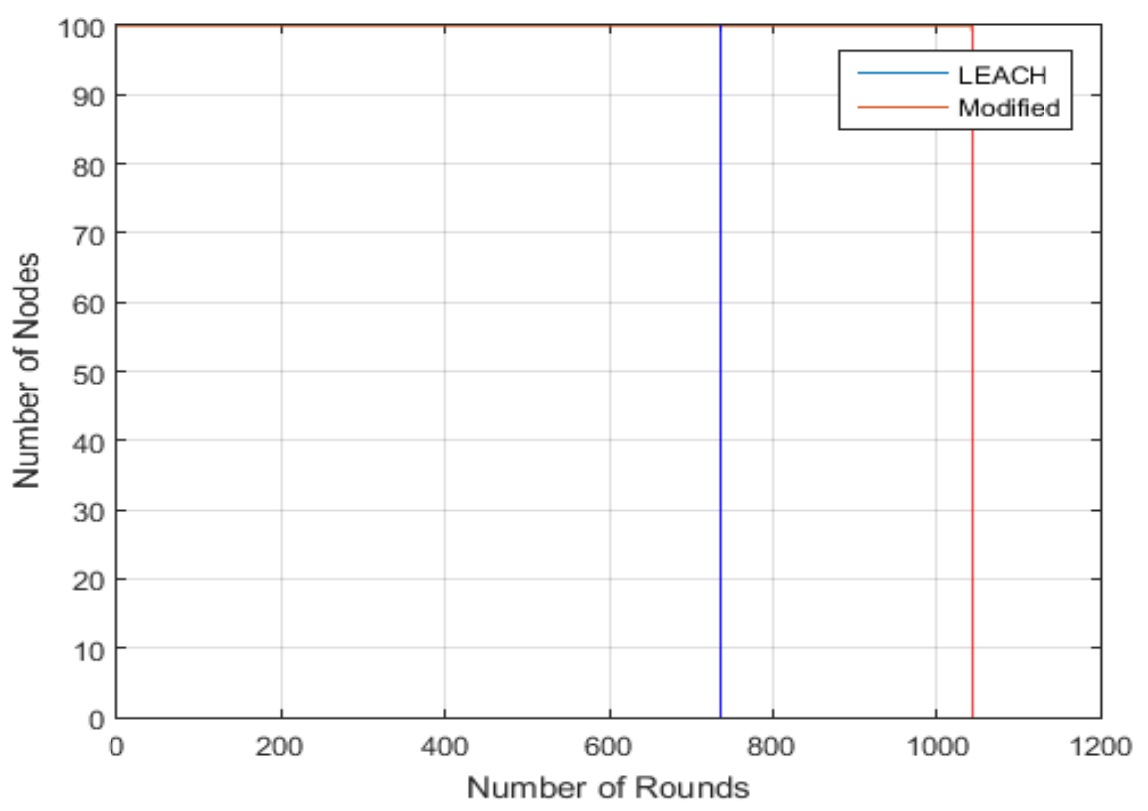

Fig (4) The Network life Time

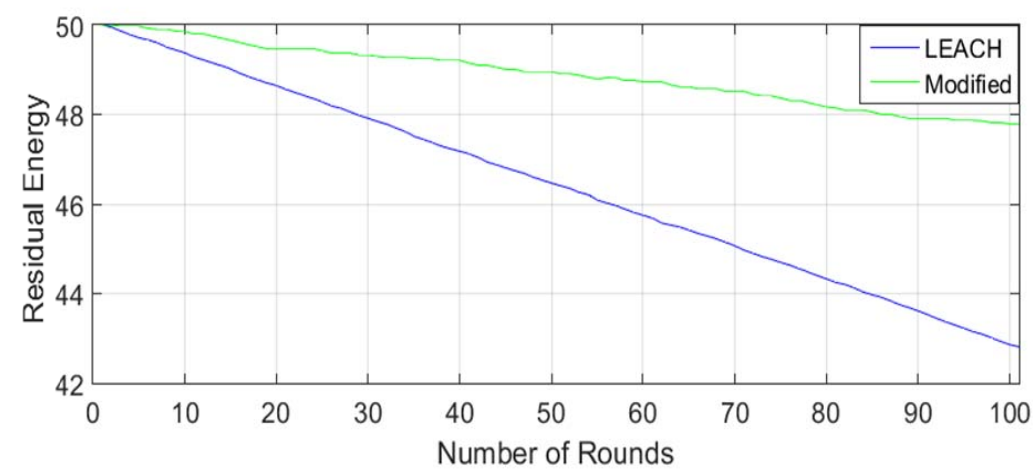

Fig (5) The Residual Energy after 100 rounds 


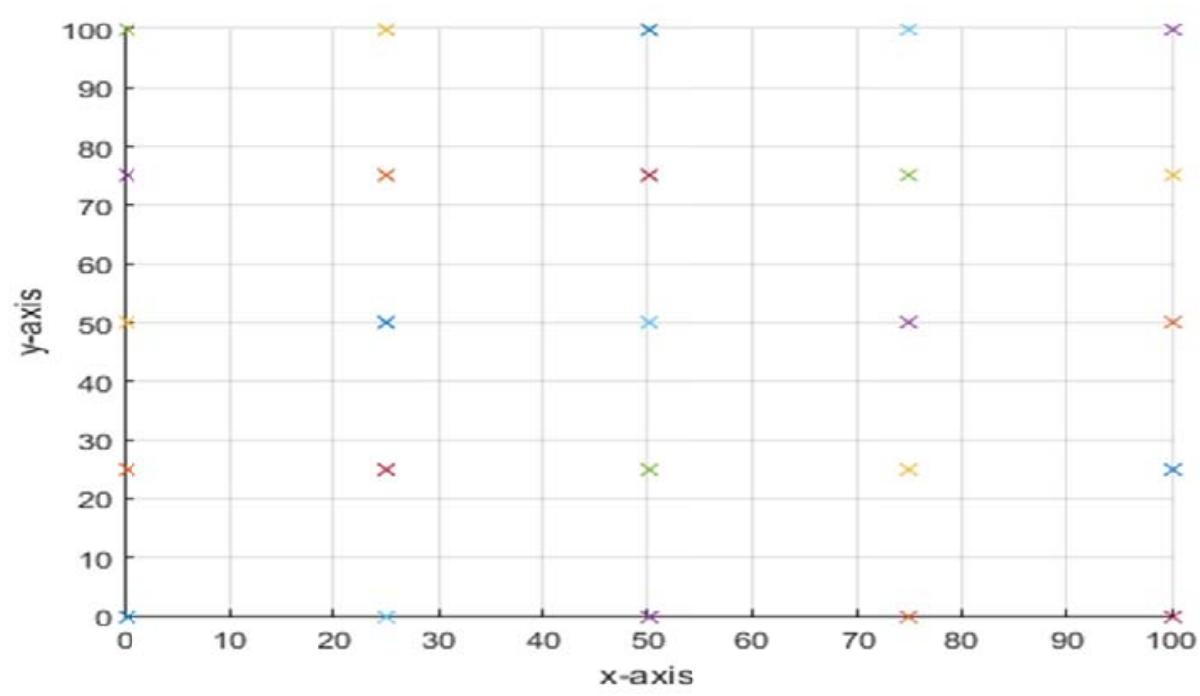

Fig (6) Location of Base Station

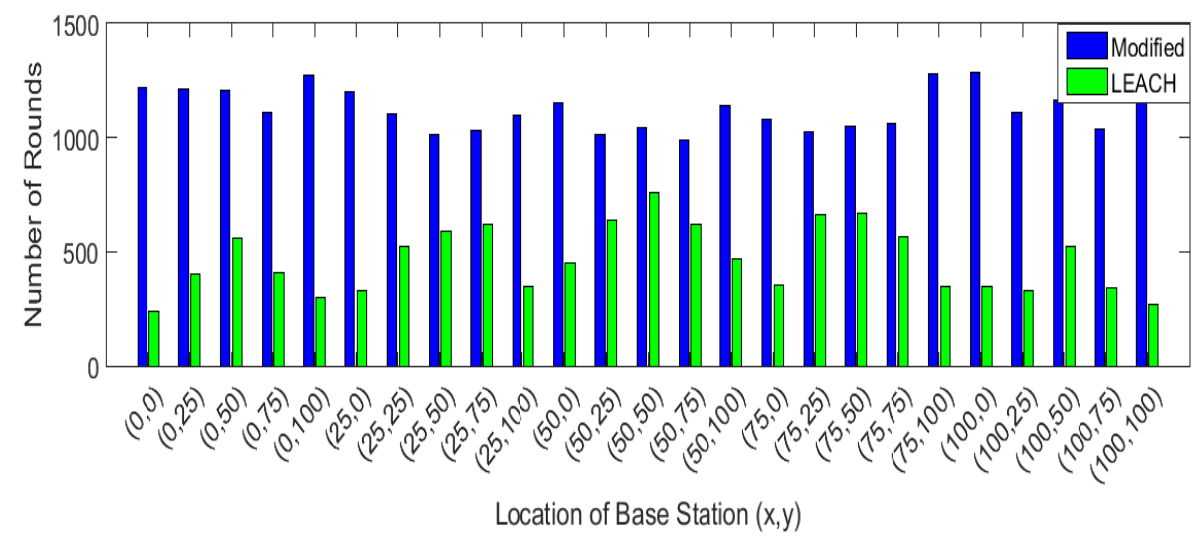

Fig (7) Lifetime of Network at Different Locations of Base Station

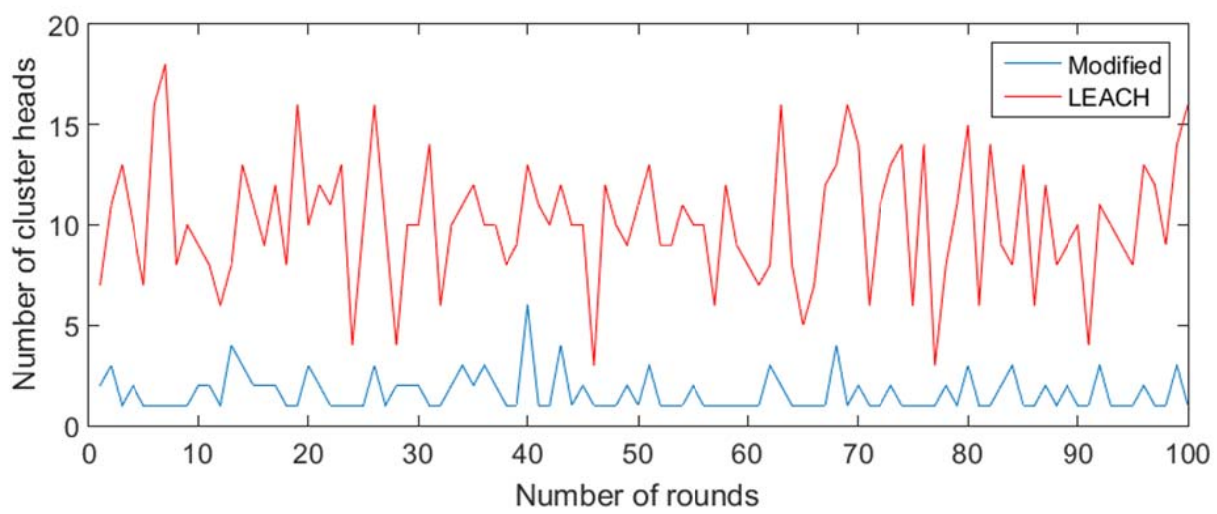

Fig (8) Number of cluster heads vs. number of rounds

Finally, the proposed algorithm (MLEACH) can perform better than the normal LEACH in view of extending the life time of a wireless sensor network irrespective of the change of location of base station. From test results the number of cluster heads are counted in each round and drawn as in fig (8).the figure shows that the number of cluster heads of MLEACH is always lower than that of the original LEACH. This will lead to an improvement in Energy consumption and hence on the lifetime of the network. The results are shown for up to 100 rounds. 


\section{CONCLUSIONS}

This paper proposed a modification for LEACH routing protocol to select cluster heads in every round based on the equation of optimized sine-cosine algorithm. Selecting a cluster head doesn't depend only on the probability of any node to be a cluster head but also on the energy of the node. Results show that the Modified LEACH (proposed algorithm) is more efficient than the normal LEACH in enlarging the lifetime of the network as a result of less consumption of energy.

\section{REFERENCES}

[1] F. L. Lewis, "Wireless sensor networks," Smart environments: technologies, protocols, and applications, pp. 11-46, 2004.

[2] C. James, "Introduction to Stochastics Search and Optimization," ed: John Wiley and Sons, Hoboken, NJ, 2003.

[3] I. BoussaïD, J. Lepagnot, and P. Siarry, "A survey on optimization metaheuristics," Information Sciences, vol. 237, pp. 82-117, 2013.

[4] W. R. Heinzelman, A. Chandrakasan, and H. Balakrishnan, "Energy-efficient communication protocol for wireless microsensor networks," in System sciences, 2000. Proceedings of the 33rd annual Hawaii international conference on, 2000, p. 10 pp. vol. 2.

[5] Y. EL FATIMI, F. Mohammed, and E. Abedellah, "Improvement of Leach Routing Algorithm Based on the Use of Game Theory and Centralized Adjustment Mechanism," International Journal of Applied Engineering Research, vol. 13, pp. 8223-8231, 2018.

[6] P. Bakaraniya and S. Mehta, "K-LEACH: An improved LEACH protocol for lifetime improvement in WSN," International Journal of Engineering Trends and Technology, vol. 4, pp. 1521-1526, 2013.

[7] F. Xiangning and S. Yulin, "Improvement on LEACH protocol of wireless sensor network," in Sensor Technologies and Applications, 2007. SensorComm 2007. International Conference on, 2007, pp. 260-264.

[8] M. S. Ali, T. Dey, and R. Biswas, "ALEACH: Advanced LEACH routing protocol for wireless microsensor networks," in Electrical and Computer Engineering, 2008. ICECE 2008. International Conference on, 2008, pp. 909-914.

[9] W. Wang, F. Du, and Q. Xu, "An improvement of LEACH routing protocol based on trust for wireless sensor networks," in Wireless Communications, Networking and Mobile Computing, 2009. WiCom'09. 5th International Conference on, 2009, pp. 1-4.

[10] J. Xu, N. Jin, X. Lou, T. Peng, Q. Zhou, and Y. Chen, "Improvement of LEACH protocol for WSN," in Fuzzy Systems and Knowledge Discovery (FSKD), 2012 9th International Conference on, 2012, pp. 2174-2177.

[11] V. Katiyar, N. Chand, G. C. Gautam, and A. Kumar, "Improvement in LEACH protocol for large-scale wireless sensor networks," in Emerging Trends in Electrical and Computer Technology (ICETECT), 2011 International Conference on, 2011, pp. 1070-1075.

[12] S. Mirjalili, "SCA: a sine cosine algorithm for solving optimization problems," Knowledge-Based Systems, vol. 96, pp. 120-133, 2016.

[13] L. M. Kamarudin, R. B. Ahmad, D. L. Ndzi, A. Zakaria, K. Kamarudin, and M. E. E. S. Ahmed, "Simulation and analysis of LEACH for wireless sensor networks in agriculture," International Journal of Sensor Networks, vol. 21, pp. 16-26, 2016.

[14] I. Dietrich and F. Dressler, "On the lifetime of wireless sensor networks, University of Erlangen," Department of Computer Science 7, Technical Report, 04/062006.

[15] M. Cardei and D.-Z. Du, "Improving wireless sensor network lifetime through power aware organization," Wireless networks, vol. 11, pp. 333-340, 2005.

[16] Y. Wang, "Topology control for wireless sensor networks," in Wireless sensor networks and applications, ed: Springer, 2008, pp. 113147. 